\title{
GALL BLADDER AGENESIS: CASE REPORT
}

Prabhu Dayal Sinwar' ${ }^{1}$ R. K. Kajla², Ramniwas Dhukiya ${ }^{3}$, Mahavir Prasad ${ }^{4}$

\section{HOW TO CITE THIS ARTICLE:}

Prabhu Dayal Sinwar, R. K. Kajla, Ramniwas Dhukiya, Mahavir Prasad. "Gall Bladder Agenesis: Case Report". Journal of Evolution of Medical and Dental Sciences 2014; Vol. 3, Issue 42, September 08; Page: 10454-10457, DOI: $10.14260 /$ jemds/2014/3361

ABSTRACT: Isolated agenesis of the gallbladder is a rare congenital anomaly that results from failure of the cystic bud to develop in utero. Patients are usually asymptomatic, and the diagnosis is commonly made as an incidental finding during abdominal surgery or at autopsy. OBJECTIVE: To aware about a rare case of Gall Bladder Agenesis in inconclusive USG findings. METHODOLOGY: Preoperative USG shows contracted gall bladder containing stone but intra-operatively GB was absent and postoperative MRCP shows absent gall bladder fossa with normal biliary radicals. RESULTS: Agenesis of the gallbladder is a rare but well-recognized congenital anomaly, the management of which is conservative. However, clinical and radiologic features mimic those of more common biliary conditions, and so patients frequently undergo unnecessary operative procedures. CONCLUSION: In conclusion, with the newer minimally invasive radiologic techniques, this situation can largely be avoided if awareness of this condition is improved. Pre-operative MRCP should be considered in cases in which ultrasound is inconclusive, and surgeons should maintain a low threshold for further investigation before any decision to operate.

KEYWORDS: MRCP, USG, Gall Bladder Fossa.

INTRODUCTION: Isolated agenesis of the gallbladder is a rare congenital anomaly that results from failure of the cystic bud to develop in utero. Since its first description by Lemery in 1701, a number of cases have been published, with a reported incidence of $0.01 \%$ to $0.06 \%{ }^{1}$

Patients are usually asymptomatic, and the diagnosis is commonly made as an incidental finding during abdominal surgery or at autopsy. ${ }^{2}$

It is estimated that $25 \%$ to $50 \%$ of patients will develop common duct stones at some point, and $23 \%$ will eventually become symptomatic, usually in the fourth or fifth decade. ${ }^{3}$

Symptoms mimic those of common biliary conditions such as cholecystitis, and routine investigations fail to distinguish between gallbladder agenesis and other conditions such as cholecystitis with cystic duct obstruction or an atrophic gallbladder. Combined with the rarity of the condition, the diagnosis is infrequently made preoperatively, and so the patient undergoes unnecessary operative intervention. Intra-operatively, the risk of iatrogenic injury is higher, and so the associated morbidity of the procedure is greater. ${ }^{4}$

Despite recent advances in biliary tract imaging, the pre-operative diagnosis of gallbladder agenesis remains elusive. Here this case report describes a case of symptomatic gallbladder agenesis with common duct stones diagnosed at laparotomy and discuss the utility of the various imaging modalities that are currently available to attempt to diagnose this condition.

CASE REPORT: A 35 years old female presented with pain right upper abdomen since 12 months at department of General Surgery, S.P. Medical College, Bikaner (Rajasthan). 
Pain is insidious onset than gradually progressive, dull aching in nature. Pain relieved by the medicines. Now pain is increases last 5-6 months. No complaint of nausea and vomiting. No complaint of fever.

- Personal History: Patient is vegetarian, Nonalcoholic, Nonsmoker, Normal bowel and bladder habits, and Normal menstrual history with regular interval.

- Past history: History of sterilization operation present $2 \frac{1}{2}$ months back.

- No history of Tuberculosis, Diabetes Mellitus, Asthma, Hypertension.

- No Drug History, No Allergic History.

General Physical Examination: Patient was conscious and oriented to time, place and person. Blood pressure - 110/70mmHg, Pulse rate - 76/min, Respiratory rate - 14/min, Temperature Afebrile, Built - Lean thin, No Icterus, No Cyanosis, No Clubbing and No any lymph- adenopathy. Pallor - Present.

Systemic Examination: Abdomen - On inspection - Scaphoid in shape.

- On palpation - Soft, no guarding, no rigidity, no tenderness, no palpable lump.

- On percussion - Tympanic note.

- On Auscultation - Normal bowel sounds present.

\section{Investigations:}

\section{Routine Blood Investigations:}

Hemoglobin - Initially $8.7 \mathrm{gm} \%$, than $10.1 \mathrm{gm} \%$ after 1 blood transfusion.

Blood Group - B+.

BT -2.10 minutes.

CT -3.30 minutes.

Blood Urea - 28mg/dl.

Serum Creatinine $-0.7 \mathrm{mg} / \mathrm{dl}$.

Fasting blood sugar $-82 \mathrm{mg} / \mathrm{dl}$.

Serum Bilirubin: Total $-1.2 \mathrm{mg} / \mathrm{dl}$, Direct $-0.3 \mathrm{mg} / \mathrm{dl}$.

SGOT - $24 \mathrm{IU} / \mathrm{l}, \mathrm{SGPT}-31 \mathrm{IU} / \mathrm{l}$, Alk. $\mathrm{PO}_{4}-170 \mathrm{IU} / \mathrm{l}$.

Serum Total Protein - 6.7gm/dl, Serum Albumin - 3.8gm/dl.

Radiological Investigations: USG Abdomen - On 23.07.13 USG indicate Contracted gall bladder and advised to rescan in fasting coming morning. On 24.07.13 Revised in fasting for gall bladder. Gall bladder is still contracted and GB area shows DAS suggesting? Chronic cholicystitis with cholelithiasis. CBD is mildly dilated $(7 \mathrm{~mm})$.

Treatment: Plan for laparoscopic cholecystectomy.

Intra-operative findings: Gall bladder not seen in GB fossa and dilated CBD present.

Laparoscopic converted in open cholecystectomy. 


\section{CASE REPORT}

Intra-operative Findings: Gall bladder not seen in all accessible areas around the liver and biliary drainage. CBD was dilated.

CBD exploration done with T-tube placement.

Postoperative Radiological Investigations: T-tube cholangiogram: IHBR, CHD, CBD well outlined by contrast. No Intraluminal filling defect and extrinsic compression seen. Free fluid of contrast in duodenum seen.

MRCP and MRI of Upper Abdomen: GB is not visualized. Drainage tube is seen in CBD.

Outcome: T-tube removed on $15^{\text {th }}$ postoperative day. Patient was discharged on $16^{\text {th }}$ post-operative day without any significant complaint.

Follow up: Patient comes in OPD with regular interval without any significant complaint.

DISCUSSION: The liver, gallbladder, and biliary system begin to develop early in the fourth week of intrauterine life as a ventral outgrowth from the caudal part of the foregut. This hepatic diverticulum divides into two parts as it grows, one representing the primordium of the liver, and the other, the primordium of the gallbladder and cystic duct. By the seventh week, vacuolation occurs, and the gallbladder and cystic duct develop a lumen. Failure of this developmental process at any stage results in agenesis of the gallbladder. ${ }^{5}$

Whereas, inappropriate migration of the gallbladder primordium will result in an ectopic gallbladder. Potential sites of ectopic gallbladder are intra-hepatic, left-sided, beneath the posterior inferior surface of liver, between the leaves of the lesser omentum, within the falciform ligament, retroperitoneal, retrohepatic, or in the retropancreatic and retroduodenal areas. ${ }^{6}$

Management options for this symptomatic group include smooth muscle relaxants, and if this fails, sphincterotomy. ${ }^{7}$

Importantly, laparotomy is not indicated if this benign condition is diagnosed pre-operatively.

Therefore, if it is diagnosed pre-operatively, the patient is spared operative intervention.

In our case, due to inconclusive USG the diagnosis was not made preoperatively, and the patient underwent cholecystectomy for presumed gallstones.

This highlights the need for greater appreciation of agenesis of the gallbladder as a cause of biliary symptoms, especially when initial USG suggest an inconclusive finding.

We should go for further confirmative radiological investigations in form of MRCP, CECT and ERCP.

However, failure of the currently available imaging modalities to differentiate accurately between agenesis of the gallbladder and other biliary diseases, combined with the lack of awareness of this condition, has meant that the majority of patients undergo surgery, with its associated morbidity.

CONCLUSION: In conclusion, agenesis of the gallbladder is a rare but well-recognized congenital anomaly, the management of which is conservative. 
However, clinical and radiologic features mimic those of more common biliary conditions, and so patients frequently undergo unnecessary operative procedures.

With the newer minimally invasive radiologic techniques, this situation can largely be avoided if awareness of this condition is improved.

Pre-operative MRCP should be considered in cases in which ultrasound is inconclusive, and surgeons should maintain a low threshold for further investigation before any decision to operate.

\section{REFERENCES:}

1. Bennion RS, Thompson JE, Tompkin RK: Agenesis of the gallbladder without extrahepatic biliary atresia. Arch Surg 1988, 123: 1257-1260.

2. Kabiri H, Domingo OH, Tzarnas CD: Agenesis of the gallbladder. Curr Surg 2006, 63: 104-106.

3. Peloponissios N, Gillet M, Cavin R, Halkic N: Agenesis of the gallbladder: a dangerously misdiagnosed malformation. World J Gastroenterol 2005, 11: 6228-6231.

4. Cabajo CM, Martin delOlmo JC, Blanco AJ, Atienza SR: Gallbladder and cystic duct absence: an infrequent malformation in laparoscopic surgery. SurgEndosc 1997, 11: 483-484.

5. Turkle SB, Swanson V, Chandrasoma P: Malformations associated with congenital absence of the gallbladder. J Med Genet 1983, 20: 445-449.

6. Sherson ND: The absent adult gallbladder. Aust N Z J Surg 1970, 39: 225-261.

7. Chopra PJ, Hussein SS: Isolated agenesis of the gallbladder. Saudi Med J 2003, 24: 409-410.

\section{AUTHORS:}

1. Prabhu Dayal Sinwar

2. R. K. Kajla

3. Ramniwas Dhukiya

4. Mahavir Prasad

\section{PARTICULARS OF CONTRIBUTORS:}

1. Senior Resident, Department General Surgery, Sardar Patel Medical College, Bikaner.

2. Associate Professor, Department of General Surgery, Sardar Patel Medical College, Bikaner.

3. Senior Resident, Department General Surgery, Sardar Patel Medical College, Bikaner.
4. Senior Resident, Department of General Surgery, Sardar Patel Medical College, Bikaner.

\section{NAME ADDRESS EMAIL ID OF THE CORRESPONDING AUTHOR:}

Dr. Prabhu Dayal Sinwar

S/o Mohan Ram,

V.P.O. Sukhwasi,

Tehsil and District,

Nagaur, Rajasthan-341001.

Email: prabhusinwar@gmail.com

Date of Submission: 21/08/2014.

Date of Peer Review: 22/08/2014.

Date of Acceptance: 01/09/2014.

Date of Publishing: 05/09/2014. 